\title{
Evaluation of a Phone Call Reminder Strategy in the Surveillance of Patients with Gastric Precancerous Lesions Lost to Follow-Up
}

\author{
Nicolas Chapelle ${ }^{a-c}$ Iva Jirka ${ }^{a}$ Matthieu Péron ${ }^{a, b}$ \\ Lucille Quénéhervé $^{a-c}$ Estelle Cauchin ${ }^{a, c}$ Yann Touchefeu ${ }^{a, c}$ \\ Emmanuel Coron ${ }^{a-c}$ Jean-François Mosnier ${ }^{b}, d$ \\ Tamara Matysiak-Budnik ${ }^{a-c}$ \\ ${ }^{a} \mathrm{CHU}$ de Nantes, Hôtel Dieu, Institut des Maladies de I'Appareil Digestif, Nantes, France; \\ bUniversité de Nantes, Nantes, France; 'INSERM, U1235, Nantes, France; dService d'Anatomie \\ Pathologique, CHU de Nantes, Nantes, France
}

\section{Keywords}

Atrophic gastritis - Early detection · Gastric cancer - Gastric precancerous lesions · Intestinal metaplasia

\begin{abstract}
Background: Surveillance of gastric precancerous lesions (GPL) may reduce gastric cancer (GC)-related mortality, but some patients with GPL are lost to follow-up. Objective: The aim of this study was to evaluate the feasibility and efficacy of a "phone-call" strategy in surveillance of the lost to follow-up patients. Patients and Methods: Among all the patients diagnosed with GPL (atrophic gastritis, intestinal metaplasia, low-grade dysplasia) between 2000 and 2015, we identified those who should undergo surveillance endoscopy according to the current guidelines. They were contacted by telephone and invited to undergo endoscopy with gastric biopsies for histological analysis. Results: Among 535 patients with GPL, 134 were contacted. Sixty-two (46\%) could not be joined, 36 did not have endoscopy for other reasons, and finally, 36 patients (22 males, median age 65 years) were included. After the median time interval of 57 months between 2 endoscopies, 18 patients showed stability, 11 regression, and 7 progression of GPL, including 1 patient who developed GC. Conclusion: Despite several telephone calls, only one-third of the contacted patients could be brought to surveillance endoscopy. Most of the patients showed stability of GPL, but 1 progressed to GC and could be successfully treated.




\section{Introduction}

Gastric cancer (GC) is one of the most common malignant diseases in the world, ranked the fifth most frequent cancer and the third most frequent cause of cancer-related death [1]. Its incidence varies widely across the world, and Europe is considered a low-incidence area [2], as compared to Asia (Japan and Korea), where the high incidence justifies a populationbased GC screening [3]. In low-incidence areas, the screening for GC or for gastric precancerous lesions (GPL) in the general population appears not cost-effective and is not recommended [4]. Nevertheless, patients with GPL, like atrophic gastritis (AG), intestinal metaplasia (IM) or dysplasia, are at higher risk of developing GC [5, 6], and European guidelines recommend endoscopic surveillance of these patients, according to the modalities that depend on the type and extension of GPL [7]. However, the adherence to the surveillance program is poor, and we and others have previously shown that only a quarter of the patients with GPL had at least one surveillance endoscopy [8]. This might be due to the patients' willingness, but also due to the physician unawareness of the guidelines, as previously shown [9, 10]. Thus, the aims of our study were (1) to evaluate the feasibility and effectiveness of a telephone call strategy to recover the lost to follow-up patients for whom surveillance was indicated according to the current guidelines and (2) to analyze the evolution of the GPL during the lost to follow-up period in these patients.

\section{Patients and Methods}

All the patients who underwent an upper endoscopy with gastric biopsies between January 1,2000, and December 31, 2015, at the University Hospital of Nantes, and in whom a GPL was diagnosed, were identified within the hospital pathology database. From these, patients over 80 and under 18 years old and the patients who did not fulfill the criteria for endoscopic surveillance according to the European guidelines [7] were excluded. In brief, the following criteria were included (for no visible lesions): for extensive AG or IM, endoscopy every 3 years, and for low-grade dysplasia (LGD), endoscopy within 12 months. After this first selection, medical files were analyzed and patients who underwent a gastric surgery, those who developed GC, and those with significant comorbidities were excluded.

The remaining patients were contacted by telephone (up to 3 calls) by 2 physicians (T.M.-B. and N.C.), and if joined, the patients were invited to undergo a surveillance upper endoscopy. In those who accepted the invitation, an upper endoscopy was performed, during which at least 5 random gastric biopsies were obtained ( 2 from the antrum, 2 from the corpus, 1 from the incisura) and sent for histopathological examination [11]. Gastritis was classified and graded according to the Sydney system [12], and dysplasia was evaluated according to Riddell's classification [13]. Helicobacter pylori was detected by May-Grunwald-Giemsa staining and, in cases of previous eradication treatment, by immunohistochemical staining with anti- $H$. pylori antibodies.

In this study, the initial endoscopy corresponds to the last endoscopy before the recall and the final endoscopy to the one performed after the recall. Histological diagnosis was compared between the initial and the final endoscopy.

\section{Results}

\section{Patients}

Among the 535 patients with GPL identified between 2000 and 2015, 401 were excluded from the study because of the following reasons: 54 were dead at the time of the study, 265 were over 80 years old, 1 was under 18 years old, 31 did not fulfill the criteria for surveillance endoscopy according to the European guidelines, 10 had a history of bariatric surgery, 34 had severe comorbidities, and 6 were incarcerated.

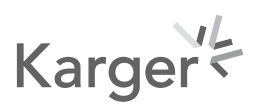




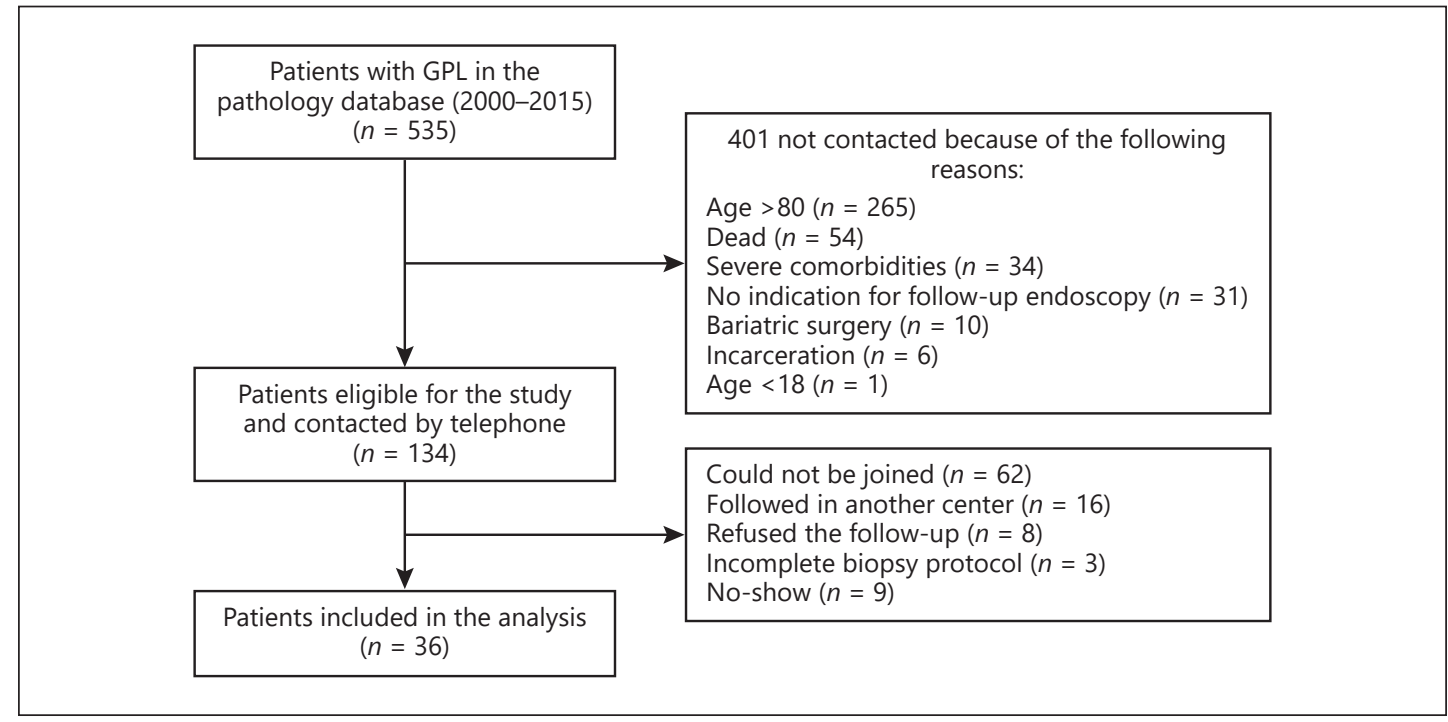

Fig. 1. Flow chart of the patients with gastric precancerous lesions (GPL) identified within the pathology database. From 535 patients with GPL initially identified, 134 were eligible for the study and were contacted by telephone. From these, 36 were finally included in the analysis.

From the 134 eligible patients, 62 (46\%) could not be joined by telephone despite at least 3 attempts. Of the 72 patients who could be joined by telephone, 16 appeared to be followed in another center, 8 refused endoscopy despite being informed about its importance, 9 agreed to have an appointment but never came (at least 2 missed appointments), and 3 had an incomplete biopsy protocol. No-show rate was 18.8\% (9/48 patients) (Fig. 1).

Thus, finally, 36 out of 134 eligible patients (31\%) were included in the analysis. Demographic and clinical characteristics of these patients are summarized in Table 1.

\section{Histological Features at the Initial and Final Endoscopy}

Initial Endoscopy

Among the 36 patients, 10 had their initial endoscopy under general anesthesia and 26 without sedation. The distribution of the most severe GPL was: AG alone: 2 patients, IM: 28 patients (including 4 patients with extensive, antrum, and corpus IM, 8 patients with corpuslimited IM, and 16 patients with antrum-limited IM), and LGD: 6 patients. Nine patients (25\%) were $H$. pylori-positive by histology, and all these patients received $H$. pylori eradication treatment.

There was no endoscopic or surgical resection at the initial endoscopy, except for 1 patient in whom endoscopic resection of an adenoma with LGD was performed. All the other patients had histologic lesions in flat mucosa (Paris 0-IIa or 0-IIb).

\section{Final Endoscopy}

The most severe GPL found at the final (post-recall) endoscopy was as follows: IM in 24 patients (antrum-limited, corpus-limited, and extensive IM in 12, 7, and 5 patients, respectively), LGD in 4 patients, and GC in 1 patient. In 7 cases, no precancerous lesion was found. All patients were $H$. pylori-negative by histology.

\section{Evolution of Histology between the Initial and Final Endoscopy}

The comparison of histology between the initial and final endoscopy showed that half of the patients $(n=18)$ had similar lesions (in terms of type and extension), 4 patients had a 
Gastrointestinal

Tumors

Table 1. Characteristics of all the patients included in the analysis $(n=36)$

\begin{tabular}{l|l}
\hline Gastrointest Tumors 2020;7:110-116 \\
\hline DOI: 10.1159/000508873 & $\begin{array}{l}\text { @ 2020 The Author(s). Published by S. Karger AG, Basel } \\
\text { www.karger.com/gat }\end{array}$ \\
\hline
\end{tabular}

Chapelle et al.: Surveillance of Gastric Precancerous Lesions

$\begin{array}{ll}\text { Males, } n(\%) & 22(61) \\ \text { Age, years, median (range) } & 65(38-79) \\ \text { Helicobacter pylori positive by histology, } n(\%) & 9(25) \\ \begin{array}{l}\text { Delay between initial and final endoscopy, months, } \\ \quad \text { median (range) }\end{array} & 57(11-121) \\ \text { Proton pump inhibitors intake, } n(\%) & 4(11) \\ \text { No symptoms, } n(\%) & 22(61) \\ \text { Epigastralgia, dyspepsia, } n(\%) & 8(22) \\ \text { GERD, } n(\%) & 6(16)\end{array}$

Initial endoscopy, the last endoscopy before recall; Final endoscopy, endoscopy after recall; GERD, gastro-esophageal reflux disease.

Table 2. Evolution of GPL between the initial and final endoscopy

\begin{tabular}{llllllll}
\hline Initial endoscopy & \multicolumn{7}{l}{ Final endoscopy (after recall) } \\
\cline { 2 - 8 } & normal & AG & $\begin{array}{l}\text { IM } \\
\text { antrum }\end{array}$ & $\begin{array}{l}\text { IM } \\
\text { corpus }\end{array}$ & $\begin{array}{l}\text { IM } \\
\text { antrum + corpus }\end{array}$ & LGD & cancer \\
\hline AG, $n=2$ & $1^{\text {a }}$ & - & - & $1^{\mathrm{c}}$ & - & - & - \\
IM (antrum only), $n=16$ & $5^{\mathrm{a}}$ & - & $8^{\mathrm{b}}$ & - & $3^{\mathrm{c}}$ & - & - \\
IM (corpus only), $n=8$ & $1^{\mathrm{a}}$ & - & $2^{\mathrm{b}}$ & $4^{\mathrm{b}}$ & $1^{\mathrm{c}}$ & - & - \\
IM (antrum + corpus), $n=4$ & - & - & - & $1^{\mathrm{a}}$ & $1^{\mathrm{b}}$ & $1^{\mathrm{c}}$ & $1^{\mathrm{c}}$ \\
LGD, $n=6$ & - & - & $2^{\mathrm{a}}$ & $1^{\mathrm{a}}$ & - & $3^{\mathrm{b}}$ & - \\
\hline
\end{tabular}

Patients marked by a superscript letter a showed a regression of GPL, those with a superscript letter $b$ showed a progression of GPL, and those with a superscript letter c showed a stability of GPL during the follow-up. GPL, gastric precancerous lesions; AG, atrophic gastritis; IM, intestinal metaplasia; LGD, low-grade dysplasia.

more severe lesion, among whom one had invasive GC, and 7 patients had no lesions (both antrum and corpus mucosa were described as normal by the pathologist). Table 2 shows the evolution of the lesions between the initial and the last endoscopy.

The patient who was diagnosed with GC could be successfully treated with surgery combined with a perioperative chemotherapy. The final histological diagnosis was an adenocarcinoma with lymphoid stroma EBV-positive staged pT2pN0. The patient is still in complete remission 2 years after surgery.

\section{Discussion}

Missed appointments and loss to follow-up are associated with increased costs and reduced healthcare efficiency and most importantly with poorer outcomes of the patients $[14,15]$. The reasons for missed appointments are multiple, lying in patients-related factors (forgetting, competition with employment and family commitments, adverse clinical experience, etc.) [16], but also in physician-related factors, mainly unawareness of the guidelines [9]. Several factors may play a role in the no-show rate for health appointments. New patients (as compared to established patients), or the patients with depression, more often tend to neglect the appointment reminder. Strategies for active recall interventions have been widely studied, mainly in the field of HIV/sexually transmissible infections, dental practice, and pediatrics, with encouraging results in terms of re-attendance/retesting rates. Several systematic 
reviews and meta-analyses reported that short message service (SMS) reminder increases appointment attendance by 14-48\% [17-19], independently of age. If there is no direct comparison between the different strategies (SMS, live call, automatic voice call, social networking sites, etc.), the studies suggest that SMS would be the most appropriate. Indeed, cell phone penetration is above $90 \%$ in the Western population, and a lot of messages can be sent automatically. A randomized controlled study reported similar efficiency between telephone call and SMS, but the latter was more cost-effective [20].

In the field of gastroenterology, in a Danish study investigating a telephone call reminder in a gastroenterology clinic, a questionnaire was sent to the no-show patients, and it turned out that the preferred reminder method was SMS (68\%), followed by a telephone call (18\%), e-mail (16\%), and letter (11\%). In that study, the telephone call reminder strategy resulted in a modest but significant $(-4 \%)$ decrease in no-show rate for appointments. Similar result was found in a recent study carried out in the USA, exploring the efficacy of a telephone call (made by a nurse), performed 7 days before the appointment for surveillance endoscopy [21]. In our study, we decided to use a live call strategy because the patients were lost to follow-up, and we believed that the need for a surveillance in GPL would be difficult to explain by SMS. Concerning the choice of the person who made the calls, we decided that the physician would be the most appropriate person to contact the patients for the first time in order to explain the need for surveillance, to reassure them, and also to judge their eligibility for surveillance (since some of them might have developed different comorbidities since they were seen for the last time). However, this strategy is time-consuming, and a literature review conducted in the field of psychiatry reported no difference in the rate of kept appointments, whether the calls were made by a physician or a behavioral health technician. These authors concluded that calls could be delegated to other health staff [22].

In our study, only half of the patients could be joined by telephone, a rate lower than the one reported in the Danish study, where $64 \%$ of the patients answered the reminder telephone call [16]. However, our study concerned the patients lost to follow-up, sometimes for several years after initial endoscopy, thus presumably many of them having been moved to another city or having changed their telephone number. Another observation was that only half of those who were successfully contacted, finally had endoscopy. This low rate may reflect a poor adherence to recommendations of the French population, as demonstrated by the high proportion of patients refusing a follow-up endoscopy despite exhaustive explanations. The no-show rate was also higher (19\%) in our study than that reported in the literature, and one of the possible explanations may be the patients' fear of the upper endoscopy, which in France is usually performed without general anesthesia.

To the best of our knowledge, this is the first study investigating active reminder strategy to recover the patients lost to endoscopic follow-up in France. Importantly, our study focused on patients with GPL, who are at increased risk for GC $[5,6]$, which is one of the most deadly cancers when diagnosed at advanced stage $[8,23]$. Since population-based screening is not recommended in low GC incidence areas like France, surveillance of patients with GPL is crucial. In this setting, the European guidelines were first published in 2012 and recently updated $[7,24]$. They provide the modalities of follow-up and surveillance, depending on the extent and type of GPL.

The studies on the evolution of the GPL remain difficult, and long-term follow-up and well-designed studies (with several gastric biopsies examined) are necessary to clarify whether some lesions can regress or not. In our series of patients, we found that in most of them, the GPL remained stable over the time, which is in agreement with the previous observations $[25,26]$. We also observed a regression of the lesions in 11 patients, including 3 with LGD. The possibility of a regression of IM and LGD, even after H. pylori eradication, is still debated. There is a risk of sampling error and variation in pathological diagnosis, especially

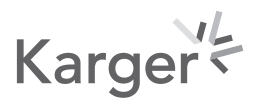


of LGD. However, in our study, this diagnosis was made in most of the cases by the same expert pathologist, which should have reduced the interobserver diagnosis variability, although the intraobserver variability may be still present. More importantly, we found in 1 patient a progression to GC, and our "phone call strategy" allowed the early diagnosis of this cancer and its curative treatment, which probably would not have been possible without this strategy since the patient was totally asymptomatic at the time of diagnosis.

In conclusion, the surveillance of patients at risk for developing GC is crucial, and lost to follow-up may have important consequences on patients' outcomes. Informing the patients on the necessity of surveillance and spreading of guidelines among physicians may surely improve the management of patients at increased risk for GC. The active "phone call strategy" allowed to recover some of these patients and even allowed to cure 1 patient with early GC, but the overall efficacy was rather low in our French population. Different tools and strategies aiming to improve patients' adherence to surveillance endoscopy need to be evaluated in the future.

\section{Statement of Ethics}

All patients have given their written informed consent for publication; the study protocol was approved by the institute's committee on human research (Comité de Protection des Personnes No. 2015-S13) and registered under the number DRC RC14_0371. The research was conducted ethically in accordance with the World Medical Association's Declaration of Helsinki.

\section{Disclosure Statement}

The authors declare that there is no conflict of interest.

\section{Funding Sources}

The study was supported by the Ligue contre le Cancer and by BIOHIT.

\section{Author Contributions}

N.C., T.M.-B., M.P., and I.J. contributed to the conception and design of the study and analysis and interpretation of data and drafted the manuscript and revised it critically. J.F.M. performed histopathological analysis of all biopsy specimens, contributed to analysis of data, and revised critically the manuscript. Y.T., E.C., L.Q., and E.C. revised critically the manuscript. All the authors approved the final version of the manuscript.

\section{References}

1 Bray F, Ferlay J, Soerjomataram I, Siegel RL, Torre LA, Jemal A. Global cancer statistics 2018: GLOBOCAN estimates of incidence and mortality worldwide for 36 cancers in 185 countries. CA Cancer J Clin. 2018;68(6): 394-424.

2 Ferlay J, Steliarova-Foucher E, Lortet-Tieulent J, Rosso S, Coebergh JW, Comber H, et al. Cancer incidence and mortality patterns in Europe: estimates for 40 countries in 2012. Eur J Cancer. 1990 2018;49(6):1374-403.

3 Pasechnikov V, Chukov S, Fedorov E, Kikuste I, Leja M. Gastric cancer: prevention, screening and early diagnosis. World J Gastroenterol. 2014;20(38):13842-62.

4 Saumoy M, Schneider Y, Shen N, Kahaleh M, Sharaiha RZ, Shah SC. Cost effectiveness of gastric cancer screening according to race and ethnicity. Gastroenterology. 2018;155(3):648-60. 
5 de Vries AC, van Grieken NC, Looman CW, Casparie MK, de Vries E, Meijer GA, et al. Gastric cancer risk in patients with premalignant gastric lesions: a nationwide cohort study in the Netherlands. Gastroenterology. 2008;134(4):945-52.

6 Song H, Ekheden IG, Zheng Z, Ericsson J, Nyrén O, Ye W. Incidence of gastric cancer among patients with gastric precancerous lesions: observational cohort study in a low risk Western population. BMJ. 2015;351:h3867.

7 Dinis-Ribeiro M, Areia M, de Vries AC, Marcos-Pinto R, Monteiro-Soares M, O'Connor A, et al. Management of precancerous conditions and lesions in the stomach (MAPS): guideline from the European Society of Gastrointestinal Endoscopy (ESGE), European Helicobacter Study Group (EHSG), European Society of Pathology (ESP), and the Sociedade Portuguesa de Endoscopia Digestiva (SPED). Endoscopy. 2012;44(1):74-94.

8 Chapelle N, Bouvier AM, Manfredi S, Drouillard A, Lepage C, Faivre J, et al. Early gastric cancer: trends in incidence, management, and survival in a well-defined French population. Ann Surg Oncol. 2016;23(11):3677-83.

9 Vance RB, Kubiliun N, Dunbar KB. How do we manage gastric intestinal metaplasia? A survey of clinical practice trends for gastrointestinal endoscopists in the United States. Dig Dis Sci. 2016;61(7):1870-8.

10 Li D, Bautista MC, Jiang SF, Daryani P, Brackett M, Armstrong MA, et al. Risks and predictors of gastric adenocarcinoma in patients with gastric intestinal metaplasia and dysplasia: a population-based study. Am J Gastroenterol. 2016;111(8):1104-13.

11 Malfertheiner P, Megraud F, O'Morain CA, Gisbert JP, Kuipers EJ, Axon AT, et al. Management of Helicobacter pylori infection-the Maastricht V/Florence Consensus Report. Gut. 2017;66(1):6-30.

12 Dixon MF, Genta RM, Yardley JH, Correa P. Classification and grading of gastritis. The updated Sydney System. International Workshop on the Histopathology of Gastritis, Houston 1994. Am J Surg Pathol. 1996;20(10): 1161-81.

13 Lauwers GY, Riddell RH. Gastric epithelial dysplasia. Gut. 1999;45(5):784-90.

14 Karter AJ, Parker MM, Moffet HH, Ahmed AT, Ferrara A, Liu JY, et al. Missed appointments and poor glycemic control: an opportunity to identify high-risk diabetic patients. Med Care. 2004;42(2):110-5.

15 Nelson EA, Maruish ME, Axler JL. Effects of discharge planning and compliance with outpatient appointments on readmission rates. Psychiatr Serv. 2000;51(7):885-9.

16 Jeppesen MH, Ainsworth MA. Telephone reminders reduced the non-attendance rate in a gastroenterology outpatient clinic. Dan Med J. 2015;62(6):

17 Guy R, Hocking J, Wand H, Stott S, Ali H, Kaldor J. How effective are short message service reminders at increasing clinic attendance? A meta-analysis and systematic review. Health Serv Res. 2012;47(2):614-32.

18 Car J, Gurol-Urganci I, de Jongh T, Vodopivec-Jamsek V, Atun R. Mobile phone messaging reminders for attendance at healthcare appointments. Cochrane Database Syst Rev. 2013;(7):CD007458.

19 Robotham D, Satkunanathan S, Reynolds J, Stahl D, Wykes T. Using digital notifications to improve attendance in clinic: systematic review and meta-analysis. BMJ Open. 2016;6(10):e012116.

20 Chen ZW, Fang LZ, Chen LY, Dai HL. Comparison of an SMS text messaging and phone reminder to improve attendance at a health promotion center: a randomized controlled trial. J Zhejiang Univ Sci B. 2008;9(1):34-8.

21 Childers RE, Laird A, Newman L, Keyashian K. The role of a nurse telephone call to prevent no-shows in endoscopy. Gastrointest Endosc. 2016;84(6):1010-e1.

22 Agarin T, Okorafor E, Kailasam V, Agarin A, Philias W, Garcia D, et al. Comparing kept appointment rates when calls are made by physicians versus behavior health technicians in Inner City Hospital: literature review and cost considerations. Community Ment Health J. 2015;51(3):300-4.

23 Allemani C, Matsuda T, Di Carlo V, Harewood R, Matz M, Nikšić M, et al. Global surveillance of trends in cancer survival 2000-14 (CONCORD-3): analysis of individual records for 37513025 patients diagnosed with one of 18 cancers from 322 population-based registries in 71 countries. Lancet Lond Engl. 2018;391:1023-75.

24 Pimentel-Nunes P, Libânio D, Marcos-Pinto R, Areia M, Leja M, Esposito G, et al. Management of epithelial precancerous conditions and lesions in the stomach (MAPS II): European Society of Gastrointestinal Endoscopy (ESGE), European Helicobacter and Microbiota Study Group (EHMSG), European Society of Pathology (ESP), and Sociedade Portuguesa de Endoscopia Digestiva (SPED) guideline update 2019. Endoscopy. 2019;51(4): 365-88.

25 den Hollander WJ, Holster IL, den Hoed CM, Capelle LG, Tang TJ, Anten MP, et al. Surveillance of premalignant gastric lesions: a multicentre prospective cohort study from low incidence regions. Gut. 2018;68(4):585-93.

26 den Hoed CM, Holster IL, Capelle LG, de Vries AC, den Hartog B, Ter Borg F, et al. Follow-up of premalignant lesions in patients at risk for progression to gastric cancer. Endoscopy. 2013;45(4):249-56. 